\title{
Biology and pathology of glia: an update
}

\author{
Werner Paulus
}

Published online: 20 December 2009

(C) Springer-Verlag 2009

We have known for decades that astrocytes are the "supportive" components in neural tissue, oligodendrocytes elaborate myelin sheaths, microglia are phagocytes, the choroid plexus produces the cerebrospinal fluid (CSF), and ependymal cells guarantee CSF flow by keeping the aqueduct open. While this extremely simplified assignment of activities is still valid today and helpful in explaining the brain to the uninitiated, the roles of different glial cell types in the brain have turned out as manifold and complex. A variety of unexpected functions have been recently proposed, such as for ependymal cells in stem cell based cell renewal, for microglia serving as a primary target in neurodegeneration, and for choroid plexus in multiple sclerosis. A steadily increasing number of studies have demonstrated extremely diverse functions of glia, and it is fair to say that there is virtually no neurological disease where glia does not play a role in pathogenesis.

The increase in the number of publications over the last 20 years differs among cell types. While the number of papers on neurons increased by $125 \%$ (from 10,040 in 1990 to 22,564 in 2009), the increase was $132 \%$ for astrocytes, $213 \%$ for oligodendrocytes, and a remarkable $1,108 \%$ for microglia (from only 125 in 1990, representing the last position at this time, to 1,385 in 2009). Interestingly, the number of papers on ependymal cells increased only slightly by $30 \%$, while there was even a $7 \%$ decrease of papers on the choroid plexus during the past 20 years (Fig. 1). The high interest in astrocytes, oligodendrocytes and especially microglia may correspond to many new (and often controversial) functions ascribed to these cell types during the past

W. Paulus $(\bowtie)$

Institute of Neuropathology, University Hospital Münster,

Domagkstr. 19, 48129 Muenster, Germany

e-mail: werner.paulus@uni-muenster.de

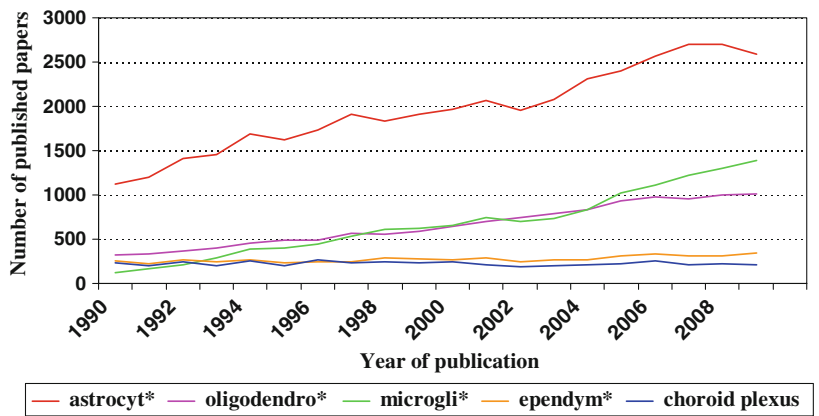

Fig. 1 Numbers of papers published per year from 1990 through 2009 dealing with glial cell types, as determined by a PubMed search on 13 December 2009 by entering the terms astrocyt*, oligodendro*, microgli*, ependym* and choroid plexus

years and/or social effects whereof neuroscientists are not exempt. The little interest in ependymal cells and the choroid plexus may be related to their restricted topographical distribution in the brain and their largely undetermined role in the pathogenesis of major neurological disorders.

This issue of Acta Neuropathologica includes five review papers on astrocytes, oligodendrocytes, ependymal cells, choroid plexus, and microglia, each of them written by experts in neurobiology and neuropathology. These papers focus on recent insight into the biology of glia, and then move on to discuss implications for neurological disease. They address cellular subtypes, molecular markers, development, ultrastructure, the molecular composition of cellular junctions and channels, activation stages, interactions with other cells, mechanisms of migration and cell death, functions in the normal brain and in a wide variety of neurological disorders, and therapeutic strategies involving glia. We hope that this cluster of review papers is useful for neurobiologists with an interest in pathology as well as for clinical neuroscientists interested in biology. 